\title{
The Pentafluorophenyl Sulfonate Ester as a Protecting Group for the Preparation of Biaryl- and Heterobiaryl-Sulfonate Esters
}

\author{
Barbara G. Avitabile ${ }^{\star, \dagger}$, Clive A. Smith and \\ Duncan B. Judd \\ ${ }^{\dagger}$ Marie Curie Post Doctoral student \\ GlaxoSmithKline, New Frontiers Science Park, Third \\ Avenue, Harlow, Essex, UK CM19 5AW \\ barbara.g.avitabile@gsk.com
}

\section{Supporting Information}

General. Reagents for synthesis were purchased from Sigma-Aldrich, Lancaster, Boron Molecular, and Strem. Anhydrous solvents were purchased from Romil. All reactions were performed under an argon atmosphere.

Medium pressure chromatography was carried out on pre-packed Isco RediSep silica cartridges, using the ISCO SQ16X instrument. Analytical (TLC) was performed using silica gel 60 (Merck, $1 \mathrm{~mm}$ ). ${ }^{1} \mathrm{H}$ and ${ }^{19} \mathrm{~F}$ NMR spectra were recorded on a Bruker $400 \mathrm{MHz}$ spectrometer and reported as chemical shifts $(\delta)$ in ppm relative to TMS, and $\mathrm{CFCl}_{3}$ respectively $(\delta=0)$. Spin multiplicities are reported as singlet (s), doublet (d), triplet (t), quartet (q), double-doublet (dd) or multiplet $(\mathrm{m})$; coupling constants $(J)$ are given in Hz. The permanent presence of solvent in analytical samples was corroborated by ${ }^{1} \mathrm{H}$ NMR spectroscopy. MP were recorded using the Perkin-Elmer Electrothermal melting point apparatus (IA6304).

4-Bromobenzenesulfonic acid pentafluorophenyl ester (2). To a solution of pentafluorophenol $(82 \mathrm{~g}, 0.445 \mathrm{~mol})$ in DCM $(3.5 \mathrm{~L})$ was added TEA $(135.09 \mathrm{~g}$, $1.335 \mathrm{~mol})$. The mixture was stirred at room temperature for 10 minutes; during which time a change of colour occurred from pale yellow to an intense orange colour indicative of the anion formation. A solution of 4-bromobenzenesulfonyl chloride (113.7g, $0.445 \mathrm{~mol})$ in DCM was then added. The reaction mixture was allowed to stir at room temperature for one hour. The solvent was evaporated 
under vacuum and the white solid was then washed with acetone and filtered; the organic filtrate was evaporated under vacuum to give a white solid.

Crystallisation from ether-hexane gave pure $2(171 \mathrm{~g}, 95 \%)$ as a white solid. TLC $\left(\mathrm{CH}_{2} \mathrm{Cl}_{2} /\right.$ hexane 1: 4): $\mathrm{R}_{f} 0.6{ }^{1} \mathrm{H}$ NMR $\left(400 \mathrm{MHz}, \mathrm{CDCl}_{3}\right): \delta 7.79\left(\mathrm{~d},{ }_{2} J=4.62\right.$ $\mathrm{Hz}, \mathrm{H}-\mathrm{C}(2 / 6), \mathrm{H}-\mathrm{C}(3 / 5)), 7.83$ (d, $2 J=4.62 \mathrm{~Hz}, \mathrm{H}-\mathrm{C}(3 / 5), \mathrm{H}-\mathrm{C}(2 / 6)) .{ }^{19} \mathrm{~F}$ NMR (400 MHz, $\left.\mathrm{CFCl}_{3}\right): \delta 150.45\left(\mathrm{~d},{ }_{2} J=6.308 \mathrm{~Hz}\right), 155.21\left(\mathrm{~d},{ }_{2} J=6.308 \mathrm{~Hz}\right)$, $160.90\left(\mathrm{~d},{ }_{2} J=6.308 \mathrm{~Hz}\right)$. MP 63.8-64.9 ${ }^{\circ} \mathrm{C}$. Analysis calc'd for $\mathrm{C}_{12} \mathrm{H}_{4} \mathrm{BrF}_{5} \mathrm{O}_{3} \mathrm{~S}: \mathrm{C}$, 35.75; H, 1.00; found: C, 35.71; H, 1.01

\section{4-Biaryl-benzenesulfonic acid pentafluorophenyl esters (4-8).}

Method A: To a solution of 4-bromobenzenesulfonic acid pentafluorophenyl ester $2(1 \mathrm{~g}, 2.48 \mathrm{mmol})$ in 1,4-dioxane $(20 \mathrm{ml})$ were added $\mathrm{Pd}\left(\mathrm{PPh}_{3}\right)_{4}(0.052 \mathrm{~g}, 0.0744 \mathrm{mmol}, 3 \mathrm{~mol} \%), \mathrm{Na}_{2} \mathrm{CO}_{3}(0.785 \mathrm{~g}, 7.44 \mathrm{mmol})$, and a solution of the boronic acid 3a-e $(2.98 \mathrm{mmol})$ in $\mathrm{EtOH}(4 \mathrm{ml})$. The reaction mixture was allowed to reflux at $104^{\circ} \mathrm{C}$ for 12 hours. The inorganic residue was filtered off on a celite pad and washed with DCM (3 x $50 \mathrm{ml})$. The organic filtrate was evaporated under vacuum giving a dark residue which was further purified by column chromatography on a gradient of $\mathrm{CH}_{2} \mathrm{Cl}_{2}$ /hexane.

Method B: To a solution of 4-bromobenzenesulfonic acid pentafluorophenyl ester $2(1 \mathrm{~g}, 2.48 \mathrm{mmol})$ in 1,4-dioxane $(5 \mathrm{ml})$ were added solid $\mathrm{Pd}\left(\mathrm{PPh}_{3}\right)_{4}(0.052 \mathrm{~g}, 0.0744 \mathrm{mmol}, 3 \mathrm{~mol} \%), \mathrm{Na}_{2} \mathrm{CO}_{3}(0.785 \mathrm{~g}, 7.44 \mathrm{mmol})$, and a solution of the boronic acid 3a-e $(2.98 \mathrm{mmol})$ in $\mathrm{EtOH}(1 \mathrm{ml})$. The reaction mixture was subjected to microwave irradiation at $180^{\circ} \mathrm{C}$ for 1 hour. The inorganic residue was filtered off on a celite pad and washed with DCM $(3 \times 50 \mathrm{ml})$. The organic filtrate was evaporated under vacuum giving a dark residue which was further purified by column chromatography on a gradient of $\mathrm{CH}_{2} \mathrm{Cl}_{2} /$ hexane.

Method C: To a solution of 4-bromobenzenesulfonic acid pentafluorophenyl ester $2(1 \mathrm{~g}, 2.48 \mathrm{mmol})$ in 1,4-dioxane $(20 \mathrm{ml})$ were added $\mathrm{Pd}\left(\mathrm{PPh}_{3}\right)_{4}(0.052 \mathrm{~g}, 0.0744 \mathrm{mmol}, 3 \mathrm{~mol} \%), \mathrm{K}_{2} \mathrm{CO}_{3}(1.03 \mathrm{~g}, 7.44 \mathrm{mmol})$, and a solution of the boronic acid 3a-e $(2.98 \mathrm{mmol})$ in $\mathrm{EtOH}(4 \mathrm{ml})$. The reaction mixture was allowed to reflux at $104^{\circ} \mathrm{C}$ for 12 hours. The inorganic residue was filtered off on a celite pad and washed with DCM $(3 \times 50 \mathrm{ml})$. The organic filtrate was evaporated under vacuum giving a dark residue which was further purified by column chromatography on a gradient of $\mathrm{CH}_{2} \mathrm{Cl}_{2} /$ hexane.

Method D: To a solution of 4-bromobenzenesulfonic acid pentafluorophenyl ester $2(1 \mathrm{~g}, 2.48 \mathrm{mmol})$ in 1,4-dioxane $(20 \mathrm{ml})$ were added $\mathrm{Pd}\left(\mathrm{PPh}_{3}\right)_{4}(0.052 \mathrm{~g}, 0.0744 \mathrm{mmol}, 3 \mathrm{~mol} \%), \mathrm{Na}_{2} \mathrm{~B}_{4} \mathrm{O}_{7}(1.5 \mathrm{~g}, 7.44 \mathrm{mmol})$, and a solution of the boronic acid 3a-e $(2.98 \mathrm{mmol})$ in $\mathrm{EtOH}(4 \mathrm{ml})$. The reaction mixture was allowed to reflux at $104^{\circ} \mathrm{C}$ for 12 hours. The inorganic residue was 
filtered off on a celite pad and washed with DCM $(3 \times 50 \mathrm{ml})$. The organic filtrate was evaporated under vacuum giving a dark residue which was further purified by column chromatography on a gradient of $\mathrm{CH}_{2} \mathrm{Cl}_{2} /$ hexane.

3',4'-Dichloro-biphenyl-4-sulfonic acid pentafluorophenyl ester (4). The derivative was synthesised using the procedure described in Method D.

The product $\mathbf{4}$ was initially purified by column chromatography using the Isco SQ16X and further crystallised from 1,4-dioxane/EtOH obtaining a white solid (0.861g, 74\%). TLC $\left(\mathrm{CH}_{2} \mathrm{Cl}_{2} /\right.$ hexane 1: 4): $\mathrm{R}_{f} 0.4{ }^{1} \mathrm{H}$ NMR $\left(400 \mathrm{MHz}, \mathrm{CDCl}_{3}\right): \delta$ $7.98\left(\mathrm{~d},{ }_{2} J=4.4 \mathrm{~Hz}, \mathrm{H}-\mathrm{C}(2 / 6), \mathrm{H}-\mathrm{C}(3 / 5)\right), 7.69$ (d, $2 J=4.2 \mathrm{~Hz}, \mathrm{H}-\mathrm{C}(3 / 5), \mathrm{H}-\mathrm{C}$ (2/6)), 7.66 (d, $\left.{ }_{1} J=1 \mathrm{~Hz}, \mathrm{H}-\mathrm{C}^{\prime}(2), \mathrm{H}-\mathrm{C}^{\prime}(6)\right), 7.52$ (d, ${ }_{1} J=4.2 \mathrm{~Hz}, \mathrm{H}-\mathrm{C}^{\prime}(5), \mathrm{H}-\mathrm{C}^{\prime}$ (6)), 7.41 (dd, $\left.1 J=4.2 \mathrm{~Hz}, \mathrm{H}-\mathrm{C}^{\prime}(6), \mathrm{H}^{-C^{\prime}}(2), J=1 \mathrm{~Hz}, \mathrm{H}-\mathrm{C}^{\prime}(6), \mathrm{H}-\mathrm{C}^{\prime}(5)\right)$ ). ${ }^{19} \mathrm{~F}$ NMR (400 MHz, $\left.\mathrm{CFCl}_{3}\right): \delta 150.46,154.98,160.70$. MP 120.4-121.2 ${ }^{\circ} \mathrm{C}$ Analysis calc'd for $\mathrm{C}_{18} \mathrm{H}_{7} \mathrm{C}_{12} \mathrm{~F}_{5} \mathrm{O}_{3} \mathrm{~S}$ : C, 46.08; $\mathrm{H}, 1.50$; found: $\mathrm{C}, 45.64 ; \mathrm{H}, 1.25$

4'-Methyl-biphenyl-4-sulfonic acid pentafluorophenyl ester (5). The derivative was synthesised using the procedure described in Method D.

The product 5 was initially purified by column chromatography using the ISCO SQ16X and further crystallised from 1,4-dioxane/EtOH obtaining a white solid (0.349g, 68\%). TLC $\left(\mathrm{CH}_{2} \mathrm{Cl}_{2} /\right.$ hexane 1: 4): $\mathrm{R}_{f} 0.4{ }^{1} \mathrm{H}$ NMR $\left(400 \mathrm{MHz}, \mathrm{CDCl}_{3}\right): \delta$ $8.03\left(\mathrm{~d},{ }_{2} J=7 \mathrm{~Hz}, \mathrm{H}-\mathrm{C}^{\prime}(2 / 6), \mathrm{H}-\mathrm{C}^{\prime}(3 / 5)\right), 7.82\left(\mathrm{~d},{ }_{2} J=6.55 \mathrm{~Hz}, \mathrm{H}-\mathrm{C}(3 / 5), \mathrm{H}-\mathrm{C}\right.$ (2/6)), $7.56\left(\mathrm{~d},{ }_{2} J=6.55 \mathrm{~Hz}, \mathrm{H}-\mathrm{C}(2 / 6), \mathrm{H}-\mathrm{C}(3 / 5)\right), 7.32$ (d, $2 J=6.31 \mathrm{~Hz}, \mathrm{H}-\mathrm{C}^{\prime}$ (3/5), H-C' (2/6)), 2.43 (s, 3). $\left.{ }^{19} \mathrm{~F} \mathrm{NMR} \mathrm{(400} \mathrm{MHz,} \mathrm{CFCl}_{3}\right)$ : $\delta 150.45,155.21$, 160.90. MP 73.8-74 ${ }^{\circ} \mathrm{C}$ Analysis calc'd for $\mathrm{C}_{19} \mathrm{H}_{11} \mathrm{~F}_{5} \mathrm{O}_{3} \mathrm{~S}: \mathrm{C}, 55.08 ; \mathrm{H}, 2.68$; found: $\mathrm{C}, 55.12 ; \mathrm{H}, 2.72$

4'-Methoxy-biphenyl-4-sulfonic acid pentafluorophenyl ester (6). The derivative was synthesised using the procedure described in Method $\mathbf{D}$.

The product 6 was initially purified by column chromatography using the Isco SQ16X and further crystallised from 1,4-dioxane/EtOH obtaining a white solid $0.446 \mathrm{~g}, 71 \%)$. TLC $\left(\mathrm{CH}_{2} \mathrm{Cl}_{2} /\right.$ hexane 1: 4): $\mathrm{R}_{f} 0.4{ }^{1} \mathrm{H} \mathrm{NMR}\left(400 \mathrm{MHz}, \mathrm{CDCl}_{3}\right): \delta$ $7.98\left(\mathrm{~d}, 2 J=8.05 \mathrm{~Hz}, \mathrm{H}-\mathrm{C}^{\prime}(2 / 6), \mathrm{H}-\mathrm{C}^{\prime}(3 / 5)\right), 7.78(\mathrm{~d}, 2 J=8.08 \mathrm{~Hz}, \mathrm{H}-\mathrm{C}(3 / 5)$, H-C (2/6)), $7.61\left(\mathrm{~d},{ }_{2} J=7.07 \mathrm{~Hz}, \mathrm{H}-\mathrm{C}(2 / 6), \mathrm{H}-\mathrm{C}(3 / 5)\right), 7.01\left(\mathrm{~d},{ }_{2} J=7.07 \mathrm{~Hz}, \mathrm{H}-\right.$ $\left.\mathrm{C}^{\prime}(3 / 5), \mathrm{H}-\mathrm{C}^{\prime}(2 / 6)\right), 3.88$ (s, 3). ${ }^{19} \mathrm{~F}$ NMR (400 MHz, $\left.\mathrm{CFCl}_{3}\right): \delta 150.45,155.21$, 160.90. MP 96.2-97.3 ${ }^{\circ} \mathrm{C}$ Analysis calc'd for $\mathrm{C}_{19} \mathrm{H}_{11} \mathrm{~F}_{5} \mathrm{O}_{4} \mathrm{~S}$ : C, 53.03; H, 2.58; found: $\mathrm{C}, 52.93 ; \mathrm{H}, 2.53$

4-Pyridin-3-yl-benzenesulfonic acid pentafluorophenyl ester (7). The derivative was synthesised using the procedure described in Method D.

The product 7 was initially purified by column chromatography using the Isco SQ16X and further crystallised from 1,4-doxane/EtOH obtaining a white solid $(0.657 \mathrm{~g}, 66 \%)$. TLC $\left(\mathrm{CH}_{2} \mathrm{Cl}_{2} /\right.$ hexane 1: 4): $\mathrm{R}_{f} 0.4{ }^{1} \mathrm{H}$ NMR $\left(400 \mathrm{MHz}, \mathrm{CDCl}_{3}\right): \delta$ 8.92 (d, ${ }_{1} J=1.57 \mathrm{~Hz}, \mathrm{H}-\mathrm{C}^{\prime}(2), \mathrm{H}-\mathrm{C}^{\prime}(6), 8.75$ (dd, ${ }_{1} J=4.52 \mathrm{~Hz}, \mathrm{H}-\mathrm{C}^{\prime}$ (3), H-C' (4), $\left.J=3.88 \mathrm{~Hz}, \mathrm{H}-\mathrm{C}^{\prime}(3), \mathrm{H}-\mathrm{C}^{\prime}(3), \mathrm{H}-\mathrm{C}^{\prime}(6)\right), 8.17$ (d, $2 J=6.75 \mathrm{~Hz}, \mathrm{H}-\mathrm{C}(2 / 6)$, 
H-C (3/5), 7.98 (dd, ${ }_{1} J=1.55 \mathrm{~Hz}, \mathrm{H}-\mathrm{C}^{\prime}(5), \mathrm{H}-\mathrm{C}^{\prime}(2), J=4.58 \mathrm{~Hz}, \mathrm{H}-\mathrm{C}^{\prime}(5), \mathrm{H}-\mathrm{C}^{\prime}$ (4)), 7.84 (d, $2 J=6.75 \mathrm{~Hz}, \mathrm{H}-\mathrm{C}(3 / 5)$, H-C (2/6)), 7.47 (m, 1). ${ }^{19} \mathrm{~F}$ NMR (400 $\left.\mathrm{MHz}, \mathrm{CFCl}_{3}\right)$ : d 150.46, 154.97, 160.78. MP 121.6-122 ${ }^{\circ} \mathrm{C}$ Analysis calc'd for $\mathrm{C}_{17} \mathrm{H}_{8} \mathrm{~F}_{5} \mathrm{NO}_{3} \mathrm{~S}$ : C, 50.88; H, 2.01; N, 3.49; found: C, 50.57; H, 1.96; N, 3.38 4-(6-Methoxy-pyridin-3-yl)-benzenesulfonic acid pentafluorophenyl ester (8). The derivative was synthesised using the procedure described in Method D. The product $\mathbf{8}$ was initially purified by column chromatography using the Isco SQ16X and further crystallised from 1,4-dioxane/EtOH obtaining a white solid (0.748g, 70\%). TLC $\left(\mathrm{CH}_{2} \mathrm{Cl}_{2} /\right.$ hexane 1: 4): $\mathrm{R}_{f} 0.4{ }^{1} \mathrm{H}$ NMR $\left(400 \mathrm{MHz}, \mathrm{CDCl}_{3}\right): \delta$ $8.48\left(\mathrm{~d},{ }_{1} J=2.09 \mathrm{~Hz}, \mathrm{H}-\mathrm{C}^{\prime}(2), \mathrm{H}-\mathrm{C}^{\prime}(5)\right), 8.08$ (d, ${ }_{2} J=5.53 \mathrm{~Hz}, \mathrm{H}-\mathrm{C}(2 / 6), \mathrm{H}-\mathrm{C}$ (3/5)), 7.84 (dd, $\left.{ }_{1} J=2.08 \mathrm{~Hz}, \mathrm{H}-\mathrm{C}^{\prime}(5), \mathrm{H}-\mathrm{C}^{\prime}(2), J=6.9 \mathrm{~Hz} \mathrm{H}-\mathrm{C}^{\prime}(5), \mathrm{H}-\mathrm{C}^{\prime}(4)\right)$, 7.77 (d, $\left.{ }_{2} J=5.55 \mathrm{~Hz}, \mathrm{H}-\mathrm{C}(3 / 5), \mathrm{H}-\mathrm{C}(2 / 6)\right), 6.90$ (d, ${ }_{1} J=6.92 \mathrm{~Hz}, \mathrm{H}-\mathrm{C}^{\prime}(4), \mathrm{H}-\mathrm{C}^{\prime}$ (5)), $4.01(\mathrm{~s}, 3) .{ }^{19} \mathrm{~F} \mathrm{NMR}\left(400 \mathrm{MHz}, \mathrm{CFCl}_{3}\right): \delta 150.46,155.17,160.89$. MP 138.3-139.5 ${ }^{\circ} \mathrm{C}$ Analysis calc'd for $\mathrm{C}_{18} \mathrm{H}_{10} \mathrm{~F}_{5} \mathrm{NO}_{4} \mathrm{~S}$ : C, 50.12; H, 2.34; N, 3.25; found: $\mathrm{C}, 50.12 ; \mathrm{H}, 2.34 ; \mathrm{N}, 3.18$ 